\title{
Study of Hysteresis Efiect between Cosmic Ray Intensity and Solar Indices
}

\section{L. Chauhanı}

Department of Physics, Govt. Model Science College

Jabalpur, M.P., INDIA

E-mail:mansukhc24@gmail.com

1Corresponding author

\section{M.K.Richhariaz}

Department of Physics, Govt. Model Science College

Jabalpur, M.P., INDIA

\section{Abstract}

In order to perform the study of cosmic ray long-term modulation, we have used six monthly averaged cosmic ray data from Oulu $(\mathrm{Rc}=$ $0.78 \mathrm{GV})$ and Rome $(\mathrm{Rc}=6.32 \mathrm{GV})$ neutron monitoring stations for three solar cycles. ( $21-23$ ) with solar activity index (sunspot numbers). The detailed analysis of hysteresis effect between these two parameters has been done. The area of hysteresis loops and time lag with correlation coefficient between cosmic ray intensity and sunspot numbers have also been calculated and noticeable differences have been found during odd and even number solar cycles. Area of odd cycle loops is much larger than even cycle loop. Each hysteresis loop consists of a small secondary loop near solar maxima. Time lag between cosmic ray intensity and sunspot numbers is different in odd and even cycles. Implication and consequences of observed differences have been discussed in this paper.

Key word: Cosmic ray intensity, sun spot numbers, time lag.

\section{Introduction:}

Correlative study between solar indices and cosmic ray intensity has been extensively studied in the past. The cause of time lag between solar activity and cosmic ray intensity and their variation has also been the matter of great interest. The 11-year solar cycle variation in cosmic ray intensity observed at Earth is anti-correlated with solar activity with some time lag. The investigation of the hysteresis phenomenon, caused by the large dimension of modulation region, between long term variation of cosmic ray intensity observed at the Earth and solar activity cycle done by [1] first and demonstrated that cosmic ray variations lagged behind sunspot activity by 6 to 12 months. The hysteresis phenomenon was analyzed by 
[2] on the basis of neutron monitor data for about one solar cycle in the frame of convectiondiffusion model of $\mathrm{CR}$ global modulation in the Heliosphere; it was shown that the dimension of the Heliosphere couldn't be smaller than $50 \mathrm{AU}$ and greater than 200-300 AU. This result is in good agreement with modern information on the possible position of terminal shock wave bounding the Heliosphere.

Investigations of cosmic ray \& solar activity hysteresis phenomenon continued by $[3,4]$ on the basis of monthly neutron monitor data.These data contain a great number of short time variation (as Forbush decrease and other events) caused by interplanetary shock waves and magnetic clouds from coronal ejection with very small time-lag (few days); this is especially important during periods of high solar activity. However, some of the recent studies of time-lag and hysteresis effect led to renewed interest in the interpretation and consequences of observed differences between time-lags in odd and even cycles as well as differences in the shape, size etc of hysteresis loops during odd and even cycles [5,6,7].

In most of earlier studies of hysteresis effect, yearly averaged value of cosmic ray intensity and solar activity index have been used. These plots show differences in features during different solar cycles. It has been found that the average time-lag between the $10.7 \mathrm{~cm}$ solar flux and cosmic ray intensity in order to decide about a suitable period over which the data may provide better insight of hysteresis phenomenon [8]. In this paper, we have studied the time-lag, correlation coefficient and hysteresis effect between long term variation in cosmic ray intensity and solar activity during the period of $21,22 \& 23_{\text {rd }}$ solar cycles.

\subsection{Data Amalysis}

In the present study we have selected cosmic ray intensity data from Oulu neutron monitoring station (cut off $=0.78 \mathrm{GV}$ ) and Rome neutron monitoring station (cut off $=6.32 \mathrm{GV}$ ) for the period of solar cycles 21,22 $\& 23$ rd (minimum solar activity expected in 2006). Hysteresis curves have been plotted between cosmic ray intensity and sunspot number (SSN) by taking six monthly mean values and the area of each hysteresis loop is calculated. Time-lag and correlation coefficient between these two parameters have also been calculated.

\subsubsection{Result and Discussion:}

We plotted the hysteresis curves for solar cycles $21,22 \& 23$ by taking six monthly mean of cosmic ray intensity and sun spot number. Fig 1 illustrates the hysteresis loops between six monthly-averaged cosmic ray intensity for Rome neutron monitoring station and SSN for SAC- 21, 22 \& 
23. These hysteresis loops show similar patterns during odd and even solar cycles. The size of each hysteresis loop is determined by calculating the area of each loop for each cycle, given in Table 1.

A small secondary loop of intensity is also observed in each hysteresis loop. This additional feature may be due to Gnevyshev gap effect- double peak structure in the maximum phase of solar activity cycles or due to peculiar particle drift effect at solar maxima. Such small secondary loops (cyclic changes) of intensity for Climax neutron monitor, superimposed at/near the solar maximum was also observed when solar polarity reversal takes place [7]. Hysteresis effects between cosmic ray intensity of low-low rigidity neutron monitoring station \& low-high rigidity station for the SAC-22 has been studied and mini loops are also obtained [12].

In fig. 2 hysteresis curves between cosmic ray intensity from Oulu station and SSN has been shown. These curves are similar in patterns as obtained for Rome station. The area of these loops is also given in the Table 1. It is clear from this table that the area of odd cycle loops are much larger than even cycle loops for both the stations. Similar odd-even asymmetry of cosmic ray cycles is observed earlier [5], such differences in behavior of cosmic ray intensity during odd and even numbered solar cycles have been discussed [9], on the basis of hypothesis that when polar magnetic field of the Sun is nearly parallel to the galactic magnetic field [10], they can easily connect, so that galactic cosmic rays, especially those of low rigidities, could enter more easily into the Heliosphere along the field lines of force, as compared to anti parallel states of the magnetic fields.

\begin{tabular}{|c|c|c|}
\hline Solar Cycle & \multicolumn{2}{|c|}{ Area } \\
\hline 21 & Rome & Oulu \\
\hline 22 & 28.8 & 63.5 \\
\hline 23 & 13.3 & 23.63 \\
\hline
\end{tabular}

Table 1: Area of hysteresis loops ( $10^{3}$ ) 

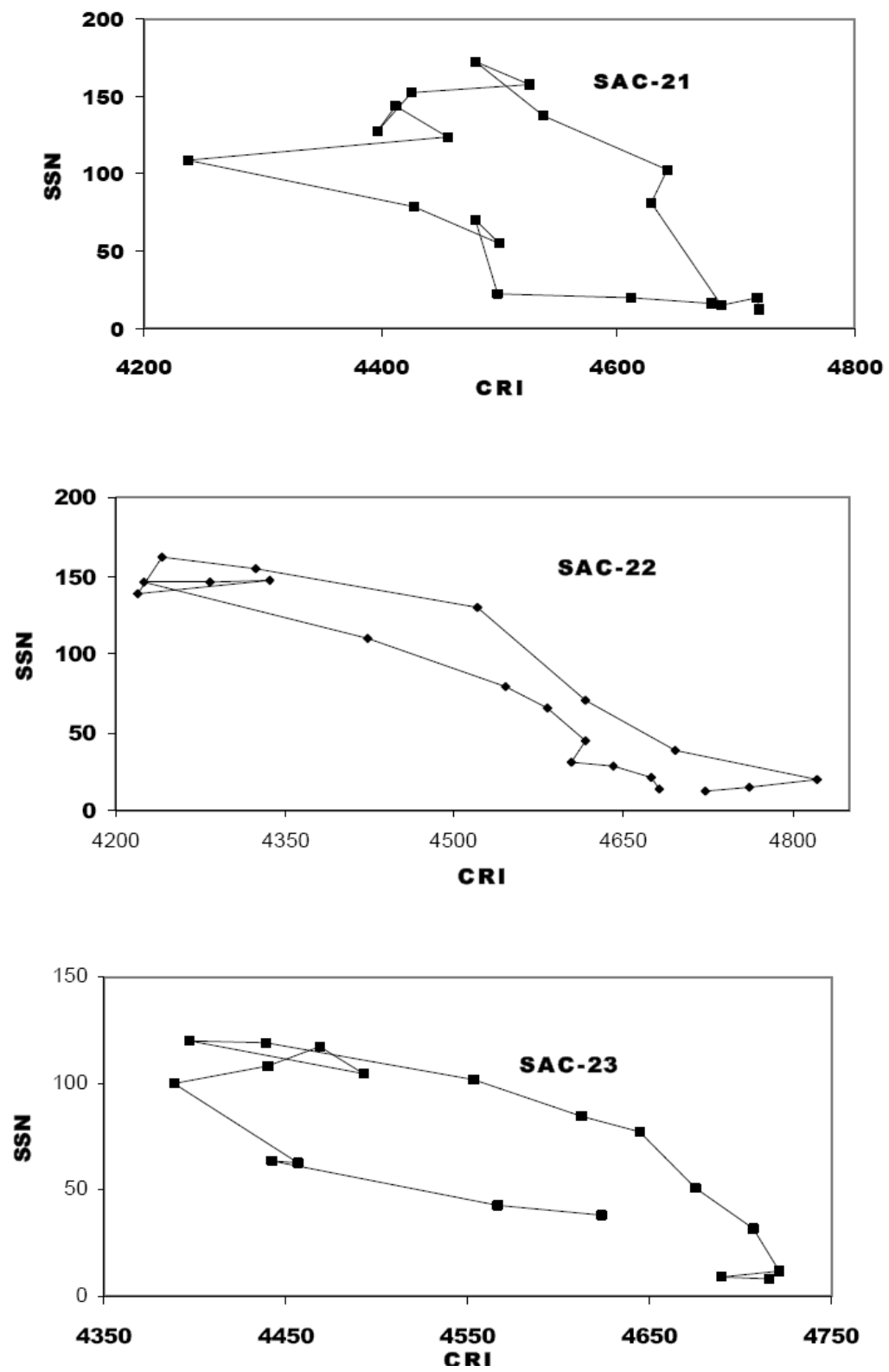

Fig 1. Hysteresis plots of six monthly-averaged cosmic ray intensity from Rome neutron monitor $(\mathrm{Rc}=6.32)$ versus sunspot number for solar cycle $21,22 \& 23$. 

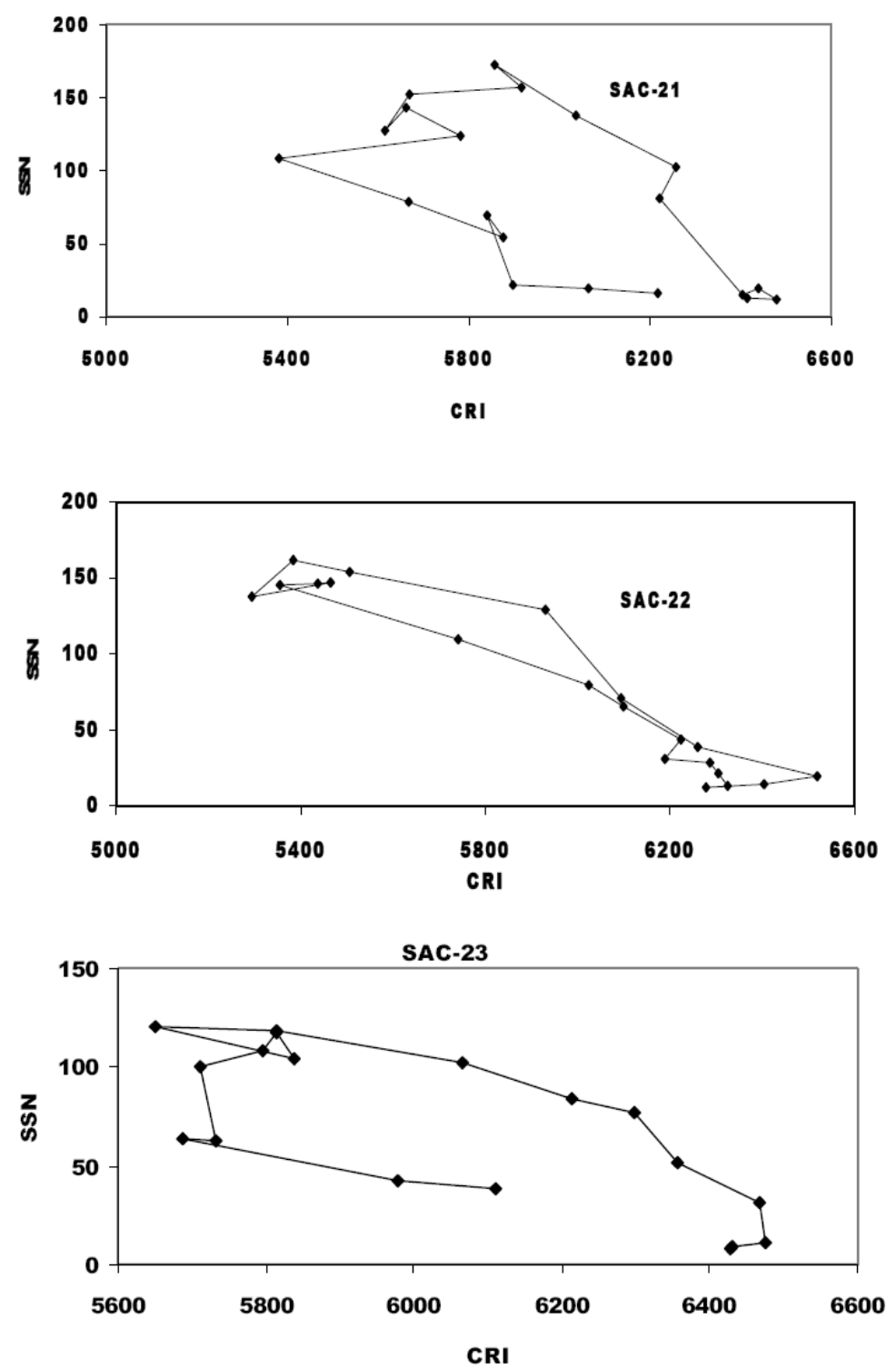

Fig 2. Hysteresis plots of six monthly-averaged cosmic ray intensity from Oulu neutron monitor versus sunspot number for solar cycle $21,22 \& 23$.

\begin{tabular}{|c|c|c|c|c|}
\hline Solar Cycle & \multicolumn{2}{|c|}{ Correlation Coefficient ${ }^{\circledR}$} & \multicolumn{2}{c|}{ Time - lag } \\
\hline & Rome & Oulu & Rome & Oulu \\
\hline $21(1976-86)$ & -0.654 & -0.647 & 6 & 7 \\
\hline $22(1986-96)$ & -0.941 & -0.955 & 9 & 8 \\
\hline $23(1996-04)$ & -0.816 & -0.816 & 6 & 6 \\
\hline
\end{tabular}

Table 2: The maximum correlation coefficient $\mathrm{R}$ between CRI and SSN with time - lag for solar cycle $21,22 \& 23$ 
Six monthly averaged values of cosmic ray intensity for Rome and Oulu neutron monitor stations and SSN has been plotted in fig 3. The maximum value of correlation coefficient between CRI and SSN for both the stations with time lags are shown in table 2 for each solar cycle separately. From table 2, one can see that the maximum value of correlation coefficient and time lag between the two parameters is different for even and odd cycles for both the stations; this shows odd-even asymmetry of cosmic ray cycles [11].

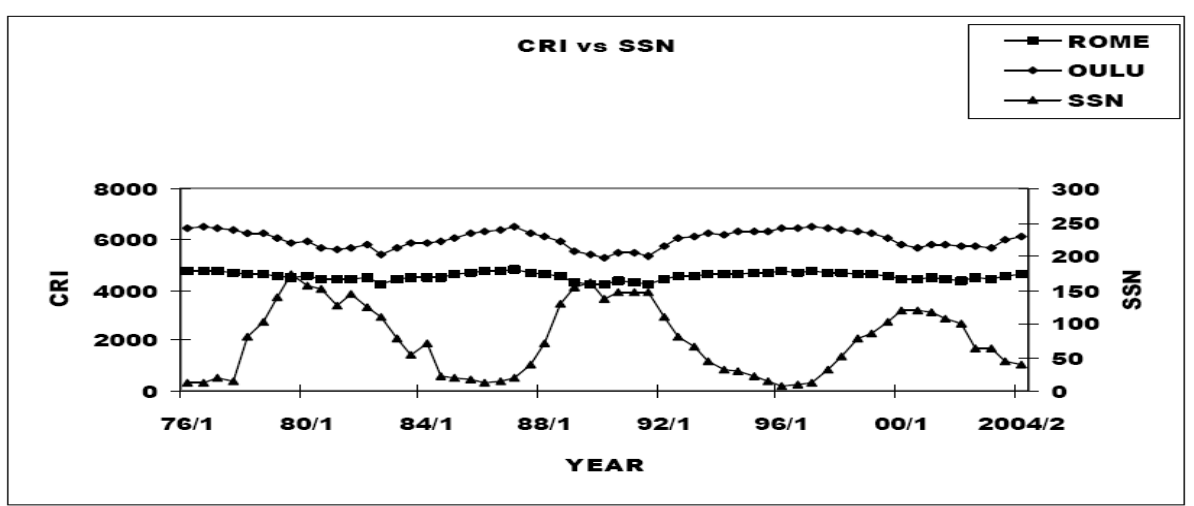

Fig (3) Variation of six monthly-averaged cosmic ray intensity for Rome and Oulu neutron monitoring stations with SSN for solar cycle 21, 22 \& 23.

\section{Conclusion}

The analysis of hysteresis loops obtained for different solar cycles shows differences between odd and even solar cycles. The area of hysteresis loops for odd solar cycles is larger than that for even cycles for both neutron monitoring stations, suggests that cosmic ray intensity behavior for even and odd solar cycles may be due to parallel and anti parallel states of solar polar magnetic field relative galactic magnetic field. A small secondary loop in each hysteresis loop may be due to peculiar particle drift effect of solar maxima. The time lag between cosmic ray intensity and sunspot number is different for odd and even solar cycles, which supports even-odd asymmetry of cosmic ray modulation. However the average time lag is estimated to be about 7 months for the period 19762004 for both the neutron monitoring stations.

\section{References:}

[1] S.E. Forbush, J.Geophys.Res.59, 525 (1954)

[2] Dorman I.V.\&Dorman L.I., Proc., 5th All Union School on Cosmo physics, Apatity P.183 (1968). 
[3] Dorman I.V et.al.,Proc. 25th ICRC, Durban, 2, 69 ( 1997a).

[4] Dorman I.V et.al.,Proc. 25th ICRC, Durban, 2, 73 (1997b).

[5] J.A. Van Allen, Geophys.Res.Lett, 27, 242453 (2000).

[6] L.I. Dorman et. al., G., Adv. Space Res. 27(3), 589 (2001).

[7] R.P. Kane, J. Geophys. Res., 108, 1379(2003).

[8] Munendra Singh, Badruddin, A.G.Ananth, 29th ICRC, Pune, 2005

[9] J.A. Otaola et.al., 19 th ICRC,La Jolla, 4, 493 (1985).

[10] H.Mavronaichalaki et. al.,Astron, Astrophycs, 330, 764 (1998).

[11] Meera Gupta, et.al., Proc 29th ICRC, Pune (2005).

[12] Laxmi Tripathi, et.al., Proc.29th ICRC, Pune (2005).

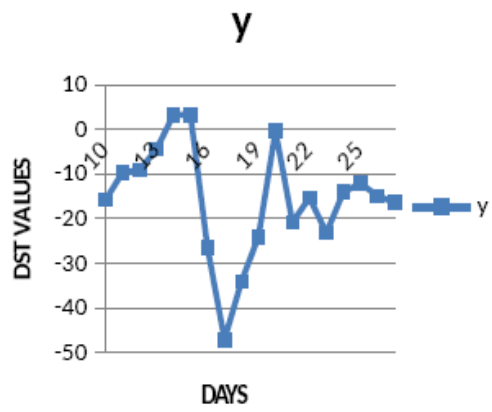

Fig.2 
The variation in Ap-index and sun spot numbers for the month of July 2017 is plotted in Fig. 3 and 4 respectively. The geomagnetic activity index Ap is found to be increased during the event period. However the rise in sun spot numbers (SSN) earlier the onset of Forbush decrease reveals that it should have some connection with the occurrence of solar flares which further affects the cosmic ray variation[5]. It is clear from fig. 3 that a drastic increase in Ap-index may be one of the causes for the large Forbush decrease of July 2017.
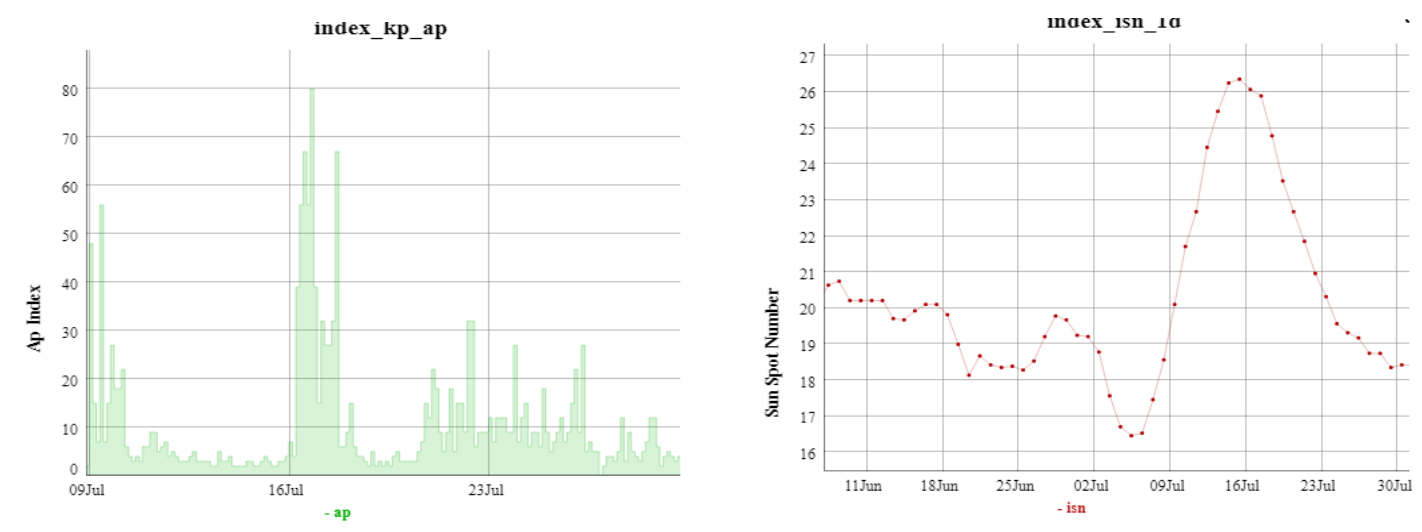

No sudden storm commencement is reported during the event time due to an intense geomagnetic activity in progress. Coronal Mass Ejections (CME's) also plays an important role in producing the Forbush decrease. However, because of non-availability of data related to CME me could not investigate its contribution.

\section{Conclusion:}


The percentage deviation in cosmic ray intensity (called Forbush decrease) for (low latitude/high altitude) neutron monitoring station shows that this decrease is more prominent in high energy particles as compared to low energy particles. Probably this decrease is a result of high activity on the sun and in the heliosphere that look place in the during July 2017. Heightened solar activities created disturbed situations in the interplanetary space that reflected in cosmic ray behavior on the background of geomagnetic storm. The Forbush decrease under investigation is probably due to the solar flare generated shockwave magnetic perturbations. However we cannot ignore the possibility of contributions by CME's. The association of Forbush decrease with geomagnetic storm needs a detailed analysis.

Ackmowledgements: The authors are indebted to various research groups working at MOSCOW neutron monitor station and the various related websites.

\section{References:}

[1] Forbush, S.E. (1938), J. Geophys. Res.43, 203.

[2] Parkar, E.N. (1963) Interplanetary dynamical process, New York.

[3] Barouch, E \& Burlaga, L.F. (1975) J. Geophys. Res.80, 449.

[4] Lockwood, J.A., Webber, W.R. \& Jokipii, J.R.(1986),

J.Geophys.Res.91, 2851.

[5] Santosh Kumar et. al., (1991) Proc.Nat.Acad.Sci., India,61(A), I. 